\title{
Explicit Finite Difference Methods for the Delay Pseudoparabolic Equations
}

\author{
I. Amirali, ${ }^{1}$ G. M. Amiraliyev, ${ }^{1}$ M. Cakir, ${ }^{2}$ and E. Cimen ${ }^{3}$ \\ ${ }^{1}$ Department of Mathematics, Faculty of Art and Science, Sinop University, 57000 Sinop, Turkey \\ ${ }^{2}$ Department of Mathematics, Faculty of Science, Yüzüncü Yil University, 65080 Van, Turkey \\ ${ }^{3}$ Department of Mathematics, Faculty of Education, Yüzüncü Yil University, 65080 Van, Turkey
}

Correspondence should be addressed to I. Amirali; ailhame@gmail.com

Received 21 August 2013; Accepted 2 December 2013; Published 4 February 2014

Academic Editors: C.-s. Liu and N. I. Mahmudov

Copyright (c) 2014 I. Amirali et al. This is an open access article distributed under the Creative Commons Attribution License, which permits unrestricted use, distribution, and reproduction in any medium, provided the original work is properly cited.

\begin{abstract}
Finite difference technique is applied to numerical solution of the initial-boundary value problem for the semilinear delay Sobolev or pseudoparabolic equation. By the method of integral identities two-level difference scheme is constructed. For the time integration the implicit rule is being used. Based on the method of energy estimates the fully discrete scheme is shown to be absolutely stable and convergent of order two in space and of order one in time. The error estimates are obtained in the discrete norm. Some numerical results confirming the expected behavior of the method are shown.
\end{abstract}

\section{Introduction}

We consider the initial-boundary value problem for pseudoparabolic equation with delay in the domain $\bar{Q}=\bar{\Omega} \cup[0, T]$; $\bar{\Omega}=[0, l], Q=\Omega \cup(0, T], \Omega=(0, l)$ :

$$
\begin{gathered}
L u:=\frac{\partial u}{\partial t}-\frac{\partial}{\partial x}\left(a(x, t) \frac{\partial^{2} u}{\partial t \partial x}\right)-\frac{\partial}{\partial x}\left(b(x, t) \frac{\partial u}{\partial x}\right) \\
=f(x, t, u(x, t), u(x, t-r)), \quad(x, t) \in Q, \\
u(x, t)=\varphi(x, t), \quad(x, t) \in \bar{\Omega} \times[-r, 0], \\
u(0, t)=u(l, t)=0, \quad t \in(0, T],
\end{gathered}
$$

where $r$ represents the delay parameter (for simplicity we assume that $T / r$ is an integer; i.e. , $T=m r$ for some integer $m>0), a(x, t) \geq \alpha>0,|b(x, t)| \leq b^{*}$, and $\varphi(x, t)$ and $f\left(x, t, u_{1}, u_{2}\right)$ are given sufficiently smooth functions satisfying certain regularity conditions in $\bar{Q}$ and $\bar{\Omega} \times[-r, 0]$ and $\bar{Q} \times \mathbb{R}^{2}$, respectively, to be specified, and furthermore

$$
\left|\frac{\partial f}{\partial u_{1}}\right| \leq c^{*}<\infty, \quad\left|\frac{\partial f}{\partial u_{2}}\right| \leq d^{*}<\infty .
$$

Equations of this type arise in many areas of mechanics and physics. Such equations are encountered, for example, as a model for two-phase porous media flows when dynamic effects in the capillary pressure are included [1-3]. They are used also to study heat conduction [4], homogeneous fluid flow in fissured rocks [5], shear in second order fluids [6-8], and other physical models. For a discussion of existence and uniqueness results of pseudoparabolic equations see $[1,9-$ 11]. Various numerical treatments of equations of this type without delay have been considered in [2,12-19] (see also the references cited in them).

In the present paper finite difference technique is applied to numerical solution of the initial-boundary value problem for the semilinear delay Sobolev or pseudoparabolic equation. By the method of integral identities with use of the piecewise linear basis functions in space and interpolating quadrature rules with weight and remainder term in integral form, two-level difference scheme is constructed (see also [12-14]) for singular perturbation cases without delay. For the time integration we use the implicit rule. The finite difference discretization is shown to be absolutely stable and convergent of order two in space and of order one in time. Based on the method of energy estimates the error analysis for approximate solution is presented. The error estimates 
are obtained in the discrete norm. Some numerical results confirming the expected behavior of the method are shown.

\section{Discretization and Mesh}

Notation. Let a set of mesh nodes that discretises $Q$ be given by

$$
\omega=\omega_{N} \times \omega_{N_{0}}
$$

with

$$
\begin{gathered}
\omega_{N}=\left\{x_{i}=i h, i=1,2, \ldots, N-1, h=\frac{l}{N}\right\}, \\
\omega_{N}^{+}=\omega_{N} \cup\left\{x_{N}=l\right\}, \quad \bar{\omega}_{N}=\omega_{N} \cup\left\{x_{0}=0, x_{N}=l\right\}, \\
\omega_{N_{0}}=\left\{t_{j}=j \tau, j=1,2, \ldots, N_{0}, \tau=\frac{T}{N_{0}}=\frac{r}{n_{0}}\right\}, \\
\bar{\omega}_{N_{0}}=\omega_{N_{0}} \cup\left\{t_{0}=0\right\}, \\
\omega_{n_{0}^{-}}=\left\{t_{j}=j \tau, j=-n_{0}, \ldots, 0\right\} .
\end{gathered}
$$

Define the following finite differences for any mesh function $v_{i}=v\left(x_{i}\right)$ given on $\bar{\omega}_{N}$ by

$$
\begin{gathered}
v_{\bar{x}, i}=\frac{v_{i}-v_{i-1}}{h}, \quad v_{x, i}=\frac{v_{i+1}-v_{i}}{h}, \\
v_{x, i}=\frac{v_{i+1}-v_{i-1}}{2 h}, \\
v_{\bar{x} x, i}=\frac{v_{x, i}-v_{\bar{x}, i}}{h}=\frac{v_{i+1}-2 v_{i}+v_{i-1}}{h^{2}} .
\end{gathered}
$$

Introduce the inner products for the mesh functions $v_{i}$ and $w_{i}$ defined on $\bar{\omega}_{N}$ as follows:

$$
\begin{gathered}
(v, w)_{0} \equiv(v, w)_{\omega_{N}}:=\sum_{i=1}^{N-1} h v_{i} w_{i}, \\
(v, w] \equiv(v, w)_{\omega_{N}^{+}}:=\sum_{i=1}^{N} h v_{i} w_{i} .
\end{gathered}
$$

For any mesh function $v_{i}$, vanishing for $i=0$ and $i=N$ we introduce the norms

$$
\begin{gathered}
\|v\|_{0}^{2} \equiv\|v\|_{0, \omega_{N}}^{2}:=(v, v)_{0}, \\
\left\|v_{\bar{x}}\right\|_{0}^{2} \equiv\left\|v_{\bar{x}}\right\|_{0, \omega_{N}^{+}}^{2}:=\left(v_{\bar{x}}, v_{\bar{x}}\right], \\
\|v\|_{1}^{2}=\|v\|_{0}^{2}+\left\|v_{\bar{x}}\right\|_{0}^{2}, \\
\|v\|_{\infty} \equiv\|v\|_{\infty, \omega_{N}}:=\max _{1 \leq i \leq N-1}\left|v_{i}\right|,
\end{gathered}
$$

and "negative" norm for any function $w_{i}(1 \leq i \leq N-1)$

$$
\|w\|_{-1}:=\sup _{v \neq 0} \frac{\left|(w, v)_{0}\right|}{\|v\|_{1}} .
$$

Given a function $g \equiv g_{i}^{j} \equiv g\left(x_{i}, t_{j}\right) ; \breve{g}=g_{i}^{(j-1)}$, defined on $\bar{\omega}$, we will also use the notation

$$
g_{\bar{t}, i}^{j}=\frac{g_{i}^{j}-g_{i}^{j-1}}{\tau_{j}}, \quad g_{t, i}^{j}=\frac{g_{i}^{j+1}-g_{i}^{j}}{\tau_{j+1}}
$$

2.1. Difference Scheme. The approach of generating difference scheme is through the integral identity

$$
\begin{gathered}
\tau^{-1} h^{-1} \int_{t_{j-1}}^{t_{j}} \int_{x_{i-1}}^{x_{i+1}} L u \psi_{i}(x) d x d t \\
=\tau^{-1} h^{-1} \int_{t_{j-1}}^{t_{j}} \int_{x_{i-1}}^{x_{i+1}} f(x, t, u(x, t), u(x, t-r)) \\
\times \psi_{i}(x) d x d t
\end{gathered}
$$

with the usual piecewise linear basis functions for the space

$$
\psi_{i}(x)= \begin{cases}h^{-1}\left(x-x_{i-1}\right), & x_{i-1}<x<x_{i} \\ h^{-1}\left(x_{i+1}-x\right), & x_{i}<x<x_{i+1}, \\ 0, & x \notin\left(x_{i-1}, x_{i+1}\right) \\ & i=1,2, \ldots, N-1 .\end{cases}
$$

Using the appropriate interpolating quadrature rules with weight and remainder term in integral form, consistent with [12-14], we obtain the precise relation

$$
\begin{aligned}
\ell u & :=u_{\bar{t}}-\left(A u_{\bar{t} \bar{x}}\right)_{x}-\left(B u_{\bar{x}}\right)_{x}+R \\
& =f(x, t-\tau, u(x, t-\tau), u(x, t-\tau-r)), \\
& (x, t) \in \omega_{N} \times \omega_{N_{0}},
\end{aligned}
$$

where

$$
\begin{aligned}
& A=\tau^{-1} h^{-1} \int_{t_{j-1}}^{t_{j}} \int_{x_{i-1}}^{x_{i}} a(x, t) d x d t, \\
& B=\tau^{-1} h^{-1} \int_{t_{j-1}}^{t_{j}} \int_{x_{i-1}}^{x_{i}} b(x, t) d x d t .
\end{aligned}
$$

The remainder term $R$ has the form

$$
R=\left(R^{(0)}\right)_{x}+R^{(1)}, \quad \text { for }(x, t) \in \omega_{N} \times \omega_{N_{0}}
$$

with

$$
\begin{aligned}
R^{(0)}= & -\tau^{-1} h^{-1} \int_{t_{j-1}}^{t_{j}} d t \int_{x_{i-1}}^{x_{i}} d x \frac{\partial a}{\partial x} \\
& \times \int_{x_{i-1}}^{x_{i}} \frac{\partial^{3} u}{\partial t \partial x^{2}}(\xi, t) K_{1}(x, \xi) d \xi
\end{aligned}
$$




$$
\begin{aligned}
& -\tau^{-1} h^{-1} \int_{t_{j-1}}^{t_{j}} d t\left\{\int_{x_{i-1}}^{x_{i}} \frac{\partial a}{\partial t}(x, t) d x\right\} \\
& \times \int_{t_{j-1}}^{t_{j}} K_{0}(\eta, t) \frac{\partial}{\partial t} u_{\bar{x}, i}(x, \eta) d \eta \\
& -\tau^{-1} h^{-1} \int_{t_{j-1}}^{t_{j}} d t \int_{x_{i-1}}^{x_{i}} d x \frac{\partial b}{\partial x}(x, t) \\
& \times \int_{x_{i-1}}^{x_{i}} \frac{\partial^{2} u}{\partial x^{2}}(\xi, t) K_{1}(x, \xi) d \xi \\
& +\tau^{-1} h^{-1} \int_{t_{j-1}}^{t_{j}} d t\left\{\int_{x_{i-1}}^{x_{i}} b(x, t) d x\right\} \\
& \times \int_{t_{j-1}}^{t_{j}} T_{0}(t-\xi) \frac{\partial}{\partial t} u_{\bar{x}, i}(x, \xi) d \xi \\
& R^{(1)}=-\tau^{-1} h^{-1} \int_{t_{j-1}}^{t_{j}} d t \int_{x_{i-1}}^{x_{i+1}} d x \varphi_{i}(x) \\
& \times \int_{x_{i-1}}^{x_{i+1}} \frac{\partial^{3} u}{\partial t \partial x^{2}}(\xi, t) K_{1}^{*}(x, \xi) d \xi \\
& +\tau^{-1} h^{-1} \int_{t_{j-1}}^{t_{j}} d t \int_{x_{i-1}}^{x_{i+1}} d x \varphi_{i}(x) \\
& \times \int_{x_{i-1}}^{x_{i+1}} \frac{d^{2}}{d x^{2}} f(\xi, t, u(\xi, t), u(\xi, t-r)) \\
& \times K_{1}^{*}(x, \xi) d \xi \\
& +\tau^{-1} \int_{t_{j-1}}^{t_{j}}\left(t_{j}-t\right) \frac{d}{d t} \\
& \times f\left(x_{i}, t, u\left(x_{i}, t\right), u\left(x_{i}, t-r\right)\right) d t, \\
& K_{1}(x, \xi)=T_{1}(x-\xi)-h^{-1}\left(x-x_{i-1}\right)\left(x_{i}-\xi\right), \\
& K_{0}(t, \eta)=T_{0}(t-\eta)-\tau^{-1}\left(t-t_{j-1}\right), \\
& K_{1}^{*}(x, \xi)=T_{1}(x-\xi)-(2 h)^{-1}\left(x-x_{i-1}\right)\left(x_{i+1}-\xi\right), \\
& T_{s}(\lambda)=\lambda^{s} / s !, \quad \lambda>0, \quad T_{s}(\lambda)=0, \quad \lambda<0 .
\end{aligned}
$$

Based on (12), we propose the following difference scheme for approximating (1):

$$
\begin{gathered}
\ell y=f(x, t-\tau, y(x, t-\tau), y(x, t-\tau-r)), \\
(x, t) \in \omega_{N} \times \omega_{N_{0}}, \\
y(0, t)=y(l, t)=0, \quad t \in \bar{\omega}_{N_{0}}, \\
y(x, 0)=\varphi(x, 0), \quad x \in \bar{\omega}_{N},
\end{gathered}
$$

where $\ell y$ is defined by (12).

\section{The Error Estimates and Convergence}

To estimate the convergence of this method, note that the error function $z=y-u$ is the solution of the discrete problem,

$$
\begin{gathered}
\ell z=f(x, t-\tau, y(x, t-\tau), y(x, t-\tau-r)) \\
-f(x, t-\tau, u(x, t-\tau), u(x, t-\tau-r))+R, \\
(x, t) \in \omega_{N} \times \omega_{N_{0}}, \\
z(x, t)=0, \quad(x, t) \in \bar{\omega}_{N} \times \omega_{n_{0}^{-}}, \\
z(0, t)=z(l, t)=0, \quad t \in \omega_{N_{0}} .
\end{gathered}
$$

Before obtaining the estimate for the solution (18) we give the following Lemma.

Lemma 1. Let the mesh function $\delta \geq 0$, defined on $\omega_{N_{0}}$, satisfy

$$
\begin{gathered}
\delta_{j} \leq \alpha+\tau \sum_{k=1}^{j}\left\{c_{1} \delta_{k-1}+c_{*} \delta_{j-n_{0}-1}+\rho_{k}\right\}, \quad j=1,2, \ldots, N_{0}, \\
\delta_{j} \leq \varphi_{j}, \quad j=-n_{0}, \ldots, 0, \quad \varphi_{0} \leq \alpha,
\end{gathered}
$$

where $0 \leq \alpha, c_{1}, c_{*}=$ const, $\rho_{j} \geq 0, \varphi_{j}$ given, $n_{0} \geq 0$ is an integer. Then

$$
\delta_{j} \leq\left(\alpha+c_{*}\|\varphi\|_{L_{1}\left(\omega_{n_{0}^{-}}\right)}\right) e^{c_{1} t_{j}}+\tau \sum_{k=1}^{j} e^{\left(c_{1}+c_{*}\right) t_{j-k}} \rho_{k}
$$

where

$$
\|\varphi\|_{L_{1}\left(\omega_{n_{0}^{-}}\right)}=\sum_{j=-n_{0}}^{0} \tau \varphi_{j}
$$

Proof. For $1 \leq j \leq n_{0}+1,-n_{0} \leq k-1-n_{0} \leq 0$ and inequality (19) reduces to

$$
\delta_{j} \leq \alpha+\tau \sum_{k=1}^{j}\left(c_{1} \delta_{k-1}+\rho_{k}\right)+c_{*}\|\varphi\|_{L_{1}\left(\omega_{n_{0}^{-}}\right)} .
$$

Applying now the difference analogue of the Gronwall's inequality we get

$$
\delta_{j} \leq\left(\alpha+c_{*}\|\varphi\|_{L_{1}\left(\omega_{n_{0}}\right)}\right) e^{c_{1} t_{j}}+\tau \sum_{k=1}^{j} e^{c_{1} t_{j-k}} \rho_{k}
$$

$$
1 \leq j \leq n_{0}+1 \text {. }
$$


For $j>n_{0}+1$, after replacing in (19) $k-n_{0}=p$, we have

$$
\begin{aligned}
\delta_{j} \leq & \alpha+\tau \sum_{k=1}^{j}\left\{c_{1} \delta_{k-1}+\rho_{k}\right\}+\tau \sum_{p=1-n_{0}}^{j-n_{0}} c_{*} \delta_{k-1} \\
= & \alpha+\tau \sum_{k=1}^{j}\left\{c_{1} \delta_{k-1}+\rho_{k}\right\} \\
& +\tau \sum_{p=1-n_{0}}^{1} c_{*} \delta_{k-1}+\tau \sum_{p=2}^{j-n_{0}} c_{*} \delta_{k-1} \\
\leq & \alpha+\tau \sum_{k=1}^{j}\left\{\left(c_{1}+c_{*}\right) \delta_{k-1}+\rho_{k}\right\} \\
& +c_{*}\|\varphi\|_{L_{1}\left(\omega_{n_{0}^{-}}\right)}
\end{aligned}
$$

which by virtue of difference analogue of the Gronwall's inequality leads to (20), immediately.

Theorem 2. Let the derivatives $\partial f / \partial t, \partial^{s} f / \partial x^{s}$, $\partial^{1+k} f / \partial u_{s} \partial x^{k}(s=1,2 ; k=0,1)$, and $\partial^{2} f / \partial u_{1}^{k} \partial u_{2}^{2-k}(k=$ $0,1,2)$ be continuous and bounded on $\bar{Q} \times \mathbb{R}^{2}, a, b \in$ $C(\bar{Q}),\left(\partial^{2} / \partial x^{2}\right) \varphi(x, t),(\partial / \partial t) \varphi(x, t) \in C(\bar{\Omega} \times[-r, 0])$, and $\partial u / \partial t, \partial u / \partial x, \partial^{2} u / \partial x^{2}$, and $\partial^{3} u / \partial x^{2} \partial t \in C(\bar{Q})$. Then for the discrete problem (17) the following error estimate holds:

$$
\|y-u\|_{1} \leq C\left(h^{2}+\tau\right), \quad t \in \omega_{N_{0}} .
$$

Proof. Consider identity

$$
\begin{aligned}
\left(\ell z, z_{\bar{t}}\right)_{\omega_{N}}=( & f\left(x, t-\tau, y\left(x, t-\tau-n_{0} \tau\right)\right) \\
& \left.-f\left(x, t-\tau, u\left(x, t-\tau-n_{0} \tau\right)\right), z_{\bar{t}}\right)_{\omega_{N}} \\
+ & \left(R, z_{\bar{t}}\right)_{\omega_{N}} .
\end{aligned}
$$

After some manipulations, we get

$$
\begin{aligned}
\left\|z_{\bar{t}}\right\|_{0}^{2}+\alpha\left\|z_{\bar{t} \bar{x}}\right\|_{0}^{2} \leq & b^{*}\left\|\breve{z}_{\bar{x}}\right\|_{0}\left\|z_{\bar{t} \bar{x}}\right\|_{0}+c^{*}\left\|z_{\bar{t}}\right\|_{0}\|\|_{0} \\
& +d^{*}\left\|z_{\bar{t}}\right\|_{0}\left\|z\left(t-\tau-n_{0} \tau\right)\right\|_{0} \\
& +\left\|z_{\bar{t} \bar{x}}\right\|_{0}\|R\|_{-1}, \\
\frac{1}{2}\left\|z_{\bar{t}}\right\|_{0}^{2}+\frac{\alpha}{2}\left\|z_{\bar{t} \bar{x}}\right\|_{0}^{2} \leq & \alpha^{-1}\left(b^{*}\right)^{2}\left\|\breve{z}_{\bar{x}}\right\|_{0}^{2}+\left(c^{*}\right)^{2}\|\bar{z}\|_{0}^{2} \\
& +\left(d^{*}\right)^{2}\left\|z\left(t-\tau-n_{0} \tau\right)\right\|_{0}^{2} \\
& +\alpha^{-1}\|R\|_{-1}^{2} .
\end{aligned}
$$

Multiplying this inequality by $\tau$ and summing it up from $k=$ 1 to $k=j$, also, using here the inequality

$$
v_{j}^{2} \leq t_{j} \tau \sum_{k=1}^{j} v_{\hat{t}, k}^{2} \leq T \tau \sum_{k=1}^{j} v_{\bar{t}, k}^{2}, \quad\left(v_{0}=0\right),
$$

we obtain

$$
\begin{aligned}
\left\|z^{j}\right\|_{0}^{2}+\alpha\left\|z_{\bar{x}}^{j}\right\|_{0}^{2} & \\
\leq & \tau \sum_{k=1}^{j}\left\{2 \alpha^{-1} T\left(b^{*}\right)^{2}\left\|\breve{z}_{\bar{x}}\right\|_{0}^{2}+2\left(c^{*}\right)^{2} T\|\breve{z}\|_{0}^{2}\right. \\
& \left.+2\left(d^{*}\right)^{2} T\|\breve{z}(t-r)\|_{0}^{2}+2 T \alpha^{-1}\|R\|_{-1}^{2}\right\} .
\end{aligned}
$$

Denoting

$$
\delta_{j}=\left\|z^{j}\right\|_{0}^{2}+\alpha\left\|z_{\bar{x}}^{j}\right\|_{0}^{2},
$$

we have

$$
\delta_{j} \leq \tau \sum_{k=1}^{j}\left\{c_{1} \delta_{k-1}+c_{*} \delta_{k-n_{0}-1}+\rho_{k}\right\}, \quad j \geqslant 1,
$$

where

$$
\begin{gathered}
c_{1}=2 T \max \left\{\alpha^{-1}\left(b^{*}\right)^{2},\left(c^{*}\right)^{2}\right\}, \quad c_{*}=2 T\left(d^{*}\right)^{2}, \\
\rho=2 T \alpha^{-1}\|R\|_{-1}^{2} .
\end{gathered}
$$

Applying now Lemma 1 we obtain

$$
\left\|z^{j}\right\|_{0}^{2}+\alpha\left\|z_{\bar{x}}^{j}\right\|_{0}^{2} \leqslant 2 T \alpha^{-1} \tau \sum_{k=1}^{j} e^{\left(c_{1}+c_{*}\right) t_{k-j}}\left\|R^{k}\right\|_{-1}^{2} .
$$

Further, in view of the fact that

$$
\begin{aligned}
\left|(R, z)_{0}\right| & \leq\left|\left(R_{x}^{(0)}, z\right)_{0}\right|+\left|\left(R^{(1)}, z\right)_{0}\right| \\
& =\left|\left(R^{(0)}, z_{\bar{x}}\right]\right|+\left|\left(R^{(1)}, z\right)_{0}\right|, \quad t \in \omega_{N_{0}},
\end{aligned}
$$

we obtain

$$
\|R\|_{-1} \leq\left\|R^{(0)}\right\|_{0, \omega_{N}^{+}}+\left\|R^{(1)}\right\|_{0, \omega_{N}}, \quad t \in \omega_{N_{0}},
$$

where $R^{(0)}$ and $R^{(1)}$ are given by (15). From (35), under the assumed smoothness, we have

$$
\tau \sum_{k=1}^{N_{0}}\left\|R^{k}\right\|_{-1}^{2}=O\left(h^{2}+\tau\right),
$$

which together with (33) completes the proof of the theorem.

Remark 3. Under sufficiently smoothness of $a(x, t)$ and $b(x, t)$ for calculations of $A$ and $B$ appropriate numerical quadrature formulae can be applied; for example, $A=a\left(x_{i}-\right.$ $\left.h / 2, t_{j}\right), A=(1 / 2)\left[a\left(x_{i}, t_{j}\right)+a\left(x_{i-1}, t_{j}\right)\right]$, and so forth.

\section{Numerical Results}

In this section, we present numerical results obtained by applying the numerical method (17) to the particular problems. 
TABLE 1: The numerical results on $(0,1) \times(0,1)$.

\begin{tabular}{|c|c|c|c|c|c|}
\hline $\begin{array}{l}\text { Nodes } \\
(x, t)\end{array}$ & Exact solution & $\begin{array}{c}\text { Numerical } \\
\text { solution } \\
h=0.02 \\
\tau=0.02 \\
\end{array}$ & $\begin{array}{l}\text { Pointwise error } \\
\qquad|y-u|\end{array}$ & $\begin{array}{c}\text { Numerical } \\
\text { solution } \\
h=0.02 \\
\tau=0.01\end{array}$ & $\begin{array}{l}\text { Pointwise error } \\
\qquad|y-u|\end{array}$ \\
\hline$(0.1,0.1)$ & $-1.570 E-02$ & $-1.571 E-02$ & $1.602 E-05$ & $-1.571 E-02$ & $8.060 E-06$ \\
\hline$(0.2,0.2)$ & $-2.759 E-02$ & $-2.765 E-02$ & $5.784 E-05$ & $-2.762 E-02$ & $2.908 E-05$ \\
\hline$(0.3,0.3)$ & $-3.558 E-02$ & $-3.570 E-02$ & $1.150 E-04$ & $-3.564 E-02$ & $5.779 E-05$ \\
\hline$(0.4,0.4)$ & $-3.976 E-02$ & $-3.994 E-02$ & $1.760 E-04$ & $-3.985 E-02$ & $8.847 E-05$ \\
\hline$(0.5,0.5)$ & $-4.033 E-02$ & $-4.056 E-02$ & $2.293 E-04$ & $-4.045 E-02$ & $1.152 E-04$ \\
\hline$(0.6,0.6)$ & $-3.757 E-02$ & $-3.783 E-02$ & $2.633 E-04$ & $-3.770 E-02$ & $1.324 E-04$ \\
\hline$(0.7,0.7)$ & $-3.180 E-02$ & $-3.207 E-02$ & $2.673 E-04$ & $-3.194 E-02$ & $1.343 E-04$ \\
\hline$(0.8,0.8)$ & $-2.338 E-02$ & $-2.362 E-02$ & $2.308 E-04$ & $-2.350 E-02$ & $1.160 E-04$ \\
\hline$(0.9,0.9)$ & $-1.267 E-02$ & $-1.281 E-02$ & $1.445 E-04$ & $-1.274 E-02$ & $7.261 E-05$ \\
\hline
\end{tabular}

TABLE 2: The numerical results on $(0,1) \times(1,2)$.

\begin{tabular}{|c|c|c|c|c|c|}
\hline $\begin{array}{l}\text { Nodes } \\
(x, t)\end{array}$ & Exact solution & $\begin{array}{c}\text { Numerical } \\
\text { solution } \\
h=0.02 \\
\tau=0.02\end{array}$ & $\begin{array}{l}\text { Pointwise error } \\
\qquad|y-u|\end{array}$ & $\begin{array}{c}\text { Numerical } \\
\text { solution } \\
h=0.02 \\
\tau=0.01\end{array}$ & $\begin{array}{l}\text { Pointwise error } \\
\qquad|y-u|\end{array}$ \\
\hline$(0.1,1.1)$ & $-5.776 E-03$ & $-5.862 E-03$ & $8.565 E-05$ & $-5.819 E-03$ & $4.203 E-05$ \\
\hline$(0.2,1.2)$ & $-1.015 E-02$ & $-1.032 E-02$ & $1.688 E-04$ & $-1.023 E-02$ & $8.481 E-05$ \\
\hline$(0.3,1.3)$ & $-1.309 E-02$ & $-1.333 E-02$ & $2.422 E-04$ & $-1.321 E-02$ & $1.217 E-04$ \\
\hline$(0.4,1.4)$ & $-1.463 E-02$ & $-1.492 E-02$ & $2.993 E-04$ & $-1.478 E-02$ & $1.504 E-04$ \\
\hline$(0.5,1.5)$ & $-1.483 E-02$ & $-1.517 E-02$ & $3.339 E-04$ & $-1.500 E-02$ & $1.678 E-04$ \\
\hline$(0.6,1.6)$ & $-1.382 E-02$ & $-1.416 E-02$ & $3.406 E-04$ & $-1.399 E-02$ & $1.711 E-04$ \\
\hline$(0.7,1.7)$ & $-1.170 E-02$ & $-1.201 E-02$ & $3.145 E-04$ & $-1.186 E-02$ & $1.580 E-04$ \\
\hline$(0.8,1.8)$ & $-8.604 E-03$ & $-8.856 E-03$ & $2.514 E-04$ & $-8.730 E-03$ & $1.263 E-04$ \\
\hline$(0.9,1.9)$ & $-4.661 E-03$ & $-4.808 E-03$ & $1.476 E-04$ & $-4.735 E-03$ & $7.415 E-05$ \\
\hline
\end{tabular}

Example 1. Consider the following linear problems:

$$
\begin{gathered}
\frac{\partial u}{\partial t}-2 \frac{\partial^{3} u}{\partial t \partial x^{2}}-\frac{\partial^{2} u}{\partial x^{2}}+u(x, t-1)=f(x, t), \\
(x, t) \in[0,1] \times[0,2] \\
u(x, t)=e^{-t}(\sinh (x)-x \sinh (1)), \\
(x, t) \in[0,1] \times[-1,0] \\
u(0, t)=0, \quad u(1, t)=0, \quad t \in(0,2],
\end{gathered}
$$

where

$$
f(x, t)=e^{-t} x \sinh (1)+e^{1-t}(\sinh (x)-x \sinh (1)) .
$$

The exact solution of this problem is $u(x, t)=$ $e^{-t}(\sinh (x)-x \sinh (1))$. The computational results are presented in Tables 1 and 2.
Example 2. Now consider the following nonlinear problem:

$$
\begin{array}{r}
\frac{\partial u}{\partial t}-3 \frac{\partial^{3} u}{\partial t \partial x^{2}}-2 \frac{\partial^{2} u}{\partial x^{2}}+\tanh (u(x, t-1))=f(x, t), \\
(x, t) \in[0,1] \times[0,2], \\
u(x, t)=e^{-t}(\cosh (x)-x \cosh (1)+x-1), \\
(x, t) \in[0,1] \times[-1,0], \\
u(1, t)=0, \quad t \in(0,2],
\end{array}
$$

where

$$
\begin{aligned}
f(x, t)= & e^{-t}(x \cosh (1)-x+1) \\
& +\tanh \left(e^{1-t}(\cosh (x)-x \cosh (1)+x-1)\right) .
\end{aligned}
$$

The exact solution of this problem is $u(x, t)=$ $e^{-t}(\cosh (x)-x \cosh (1)+x-1)$. The computational results are presented in Tables 3 and 4 . 
TABLE 3: The numerical results on $(0,1) \times(0,1)$.

\begin{tabular}{|c|c|c|c|c|c|}
\hline $\begin{array}{l}\text { Nodes } \\
(x, t)\end{array}$ & Exact solution & $\begin{array}{c}\text { Numerical } \\
\text { solution } \\
h=0.02 \\
\tau=0.02\end{array}$ & $\begin{array}{c}\text { Pointwise error } \\
\qquad|y-u|\end{array}$ & $\begin{array}{c}\text { Numerical } \\
\text { solution } \\
h=0.02 \\
\tau=0.01\end{array}$ & $\begin{array}{c}\text { Pointwise error } \\
\qquad|y-u|\end{array}$ \\
\hline$(0.1,0.1)$ & $-4.461 E-02$ & $-4.465 E-02$ & $4.503 E-05$ & $-4.463 E-02$ & $2.265 E-05$ \\
\hline$(0.2,0.2)$ & $-7.249 E-02$ & $-7.264 E-02$ & $1.490 E-04$ & $-7.257 E-02$ & $7.494 E-05$ \\
\hline$(0.3,0.3)$ & $-8.710 E-02$ & $-8.738 E-02$ & $2.736 E-04$ & $-8.724 E-02$ & $1.376 E-04$ \\
\hline$(0.4,0.4)$ & $-9.127 E-02$ & $-9.166 E-02$ & $3.893 E-04$ & $-9.146 E-02$ & $1.957 E-04$ \\
\hline$(0.5,0.5)$ & $-8.728 E-02$ & $-8.776 E-02$ & $4.741 E-04$ & $-8.752 E-02$ & $2.383 E-04$ \\
\hline$(0.6,0.6)$ & $-7.704 E-02$ & $-7.755 E-02$ & $5.115 E-04$ & $-7.730 E-02$ & $2.571 E-04$ \\
\hline$(0.7,0.7)$ & $-6.206 E-02$ & $-6.255 E-02$ & $4.896 E-04$ & $-6.231 E-02$ & $2.461 E-04$ \\
\hline$(0.8,0.8)$ & $-4.359 E-02$ & $-4.399 E-02$ & $4.003 E-04$ & $-4.379 E-02$ & $2.012 E-04$ \\
\hline$(0.9,0.9)$ & $-2.264 E-02$ & $-2.287 E-02$ & $2.381 E-04$ & $-2.276 E-02$ & $1.197 E-04$ \\
\hline
\end{tabular}

TABLE 4: The numerical results on $(0,1) \times(1,2)$.

\begin{tabular}{|c|c|c|c|c|c|}
\hline $\begin{array}{l}\text { Nodes } \\
(x, t)\end{array}$ & Exact solution & $\begin{array}{c}\text { Numerical } \\
\text { solution } \\
h=0.02 \\
\tau=0.02\end{array}$ & $\begin{array}{c}\text { Pointwise error } \\
\qquad|y-u|\end{array}$ & $\begin{array}{c}\text { Numerical } \\
\text { solution } \\
h=0.02 \\
\tau=0.01\end{array}$ & $\begin{array}{l}\text { Pointwise error } \\
\qquad|y-u|\end{array}$ \\
\hline$(0.1,1.1)$ & $-1.641 E-02$ & $-1.663 E-02$ & $2.192 E-04$ & $-1.652 E-02$ & $1.101 E-04$ \\
\hline$(0.2,1.2)$ & $-2.667 E-02$ & $-2.706 E-02$ & $3.961 E-04$ & $-2.686 E-02$ & $1.990 E-04$ \\
\hline$(0.3,1.3)$ & $-3.204 E-02$ & $-3.257 E-02$ & $5.251 E-04$ & $-3.230 E-02$ & $2.637 E-04$ \\
\hline$(0.4,1.4)$ & $-3.357 E-02$ & $-3.417 E-02$ & $6.032 E-04$ & $-3.387 E-02$ & $3.029 E-04$ \\
\hline$(0.5,1.5)$ & $-3.211 E-02$ & $-3.274 E-02$ & $6.289 E-04$ & $-3.242 E-02$ & $3.158 E-04$ \\
\hline$(0.6,1.6)$ & $-2.834 E-02$ & $-2.894 E-02$ & $6.024 E-04$ & $-2.864 E-02$ & $3.024 E-04$ \\
\hline$(0.7,1.7)$ & $-2.283 E-02$ & $-2.335 E-02$ & $5.244 E-04$ & $-2.309 E-02$ & $2.633 E-04$ \\
\hline$(0.8,1.8)$ & $-1.603 E-02$ & $-1.643 E-02$ & $3.966 E-04$ & $-1.623 E-02$ & $1.991 E-04$ \\
\hline$(0.9,1.9)$ & $-8.328 E-03$ & $-8.550 E-03$ & $2.211 E-04$ & $-8.439 E-03$ & $1.110 E-04$ \\
\hline
\end{tabular}

It can be observed that the obtained results are essentially in agreement with the theoretical analysis described above.

\section{Conclusion}

In this paper, we proposed an efficient numerical method for solving initial-boundary value problem for the semilinear pseudoparabolic equation. The proposed finite difference method was constructed, and based on the method of energy estimates the fully discrete scheme was shown to be absolutely stable and convergent of order two in space and of order one in time. The error estimates were obtained in discrete norm. Numerical results were presented, which numerically validate this theoretical result.

\section{Conflict of Interests}

The authors declare that there is no conflict of interests regarding the publication of this paper.

\section{References}

[1] Y. Fan and I. S. Pop, "A class of pseudo-parabolic equations: existence, uniqueness of weak solutions, and error estimates for the Euler-implicit discretization," Mathematical Methods in the Applied Sciences, vol. 34, no. 18, pp. 2329-2339, 2011.

[2] Y. Fan and I. S. Pop, "Equivalent formulations and numerical schemes for a class of pseudo-parabolic equations," Journal of Computational and Applied Mathematics, vol. 246, pp. 86-93, 2013.

[3] S. M. Hassanizadeh and W. G. Gray, "Thermodynamic basis of capillary pressure in porous media," Water Resources Research, vol. 29, no. 10, pp. 3389-3405, 1993.

[4] P. J. Chen and M. E. Gurtin, "On a theory of heat conduction involving two temperatures," Zeitschrift für Angewandte Mathematik und Physik, vol. 19, no. 4, pp. 614-627, 1968.

[5] G. I. Barenblatt, I. P. Zheltov, and I. N. Kochina, "Basic concepts in the theory of seepage of homogeneous liquids in fissured rocks," Journal of Applied Mathematics and Mechanics, vol. 24, no. 5, pp. 1286-1303, 1960.

[6] B. D. Coleman and W. Noll, "An approximation theorem for functionals, with applications in continuum mechanics," Archive for Rational Mechanics and Analysis, vol. 6, no. 1, pp. 355-370, 1960.

[7] R. R. Huilgol, "A second order fluid of the differential type," International Journal of Non-Linear Mechanics, vol. 3, no. 4, pp. 471-482, 1968.

[8] T. W. Ting, "Certain non-steady flows of second-order fluids," Archive for Rational Mechanics and Analysis, vol. 14, pp. 1-26, 1963. 
[9] P. L. Davis, "A quasilinear parabolic and a related third order problem," Journal of Mathematical Analysis and Applications, vol. 40, no. 2, pp. 327-335, 1972.

[10] J. H. Lightbourne III and S. M. Rankin III, "A partial functional differential equation of Sobolev type," Journal of Mathematical Analysis and Applications, vol. 93, no. 2, pp. 328-337, 1983.

[11] R. E. Showalter and T. W. Ting, "Pseudo-parabolic partial differential equations," SIAM Journal on Numerical Analysis, vol. 1, pp. 1-26, 1970.

[12] G. M. Amiraliyev and I. G. Amireliyeva, "Difference schemes for the singularly perturbed Sobolev equations," in Proceedings of the International Conference on Difference Equations, Special Functions and Applications, pp. 23-40, Munich, Germany, 2005.

[13] G. M. Amiraliyev, H. Duru, and I. G. Amiraliyeva, "A parameter-uniform numerical method for a Sobolev problem with initial layer," Numerical Algorithms, vol. 44, no. 2, pp. 185203, 2007.

[14] G. M. Amiraliyev and Y. D. Mamedov, "Difference schemes on the uniform mesh for a singularly perturbed pseudo-parabolic equations," Turkish Journal of Mathematics, vol. 19, pp. 207-222, 1995.

[15] R. E. Ewing, "Numerical solution of Sobolev partial differential equataions," SIAM Journal on Numerical Analysis, vol. 12, no. 3, pp. 345-363, 1975.

[16] R. E. Ewing, “Time-stepping Galerkin methods for nonlinear Sobolev partial differential equataions," SIAM Journal on Numerical Analysis, vol. 15, pp. 1125-1150, 1978.

[17] W. H. Ford and T. W. Ting, "Uniform error estimates for difference approximations to nonlinear pseudo-parabolic partial differential equations," SIAM Journal on Numerical Analysis, vol. 11, no. 1, pp. 155-169, 1974.

[18] H. Gu, "Characteristic finite element methods for non-linear Sobolev equations," Applied Mathematics and Computation, vol. 102, no. 1, pp. 51-62, 1999.

[19] T. Sun and D. Yang, "The finite difference streamline diffusion methods for Sobolev equations with convection-dominated term," Applied Mathematics and Computation, vol. 125, no. 2-3, pp. 325-345, 2002. 


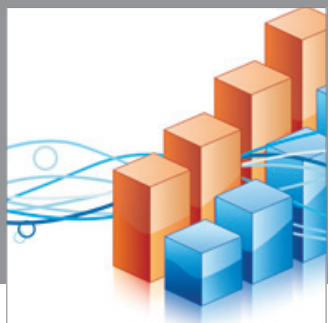

Advances in

Operations Research

mansans

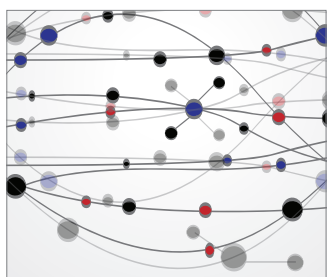

The Scientific World Journal
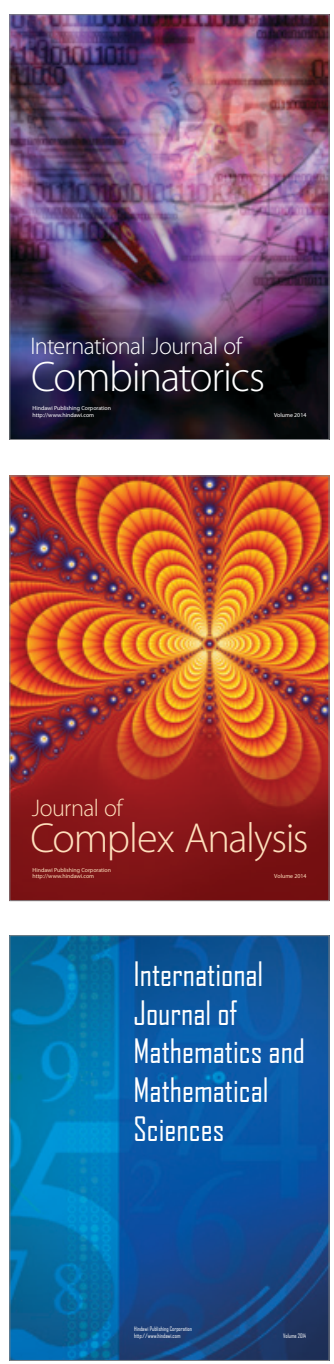
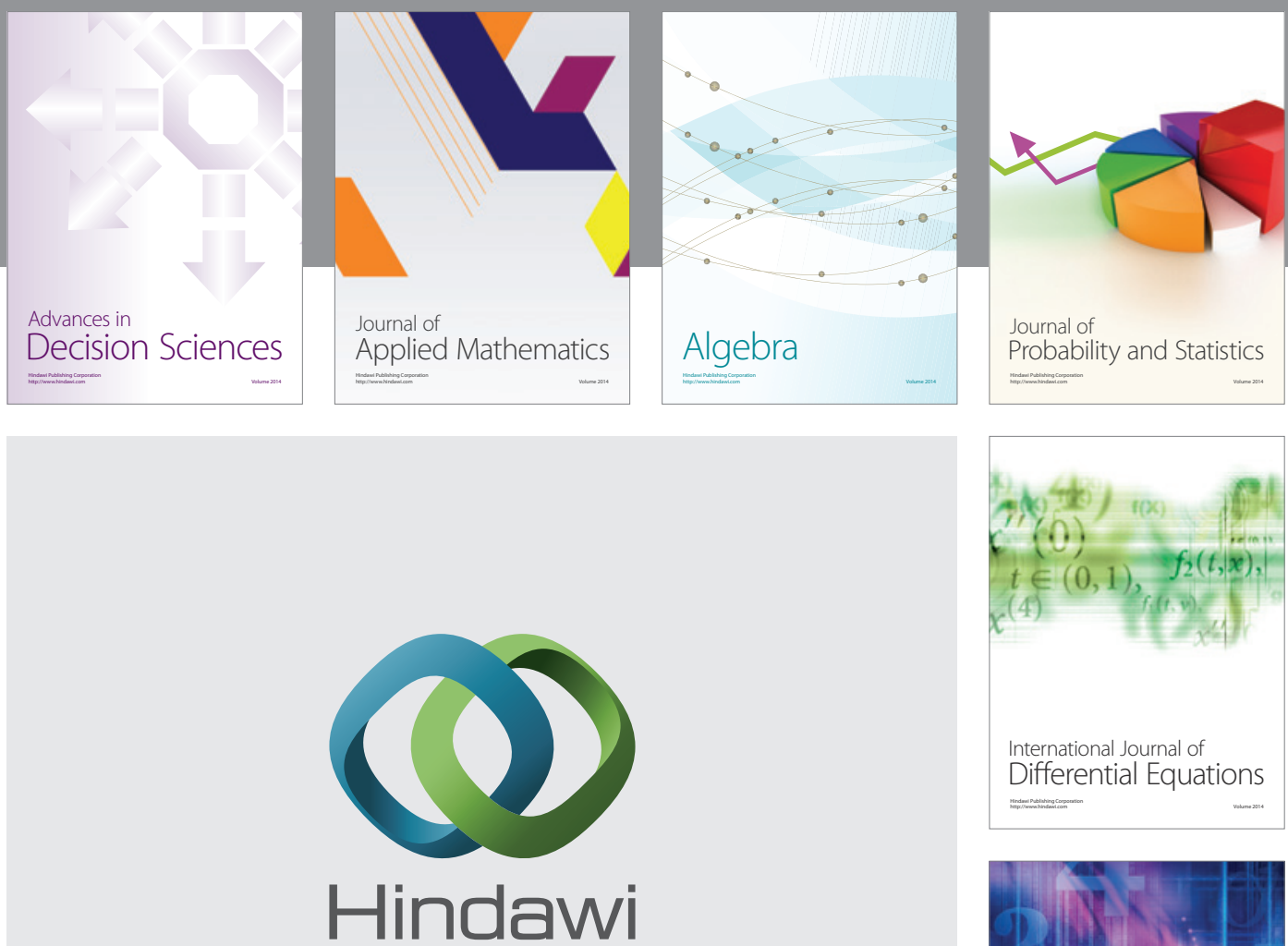

Submit your manuscripts at http://www.hindawi.com
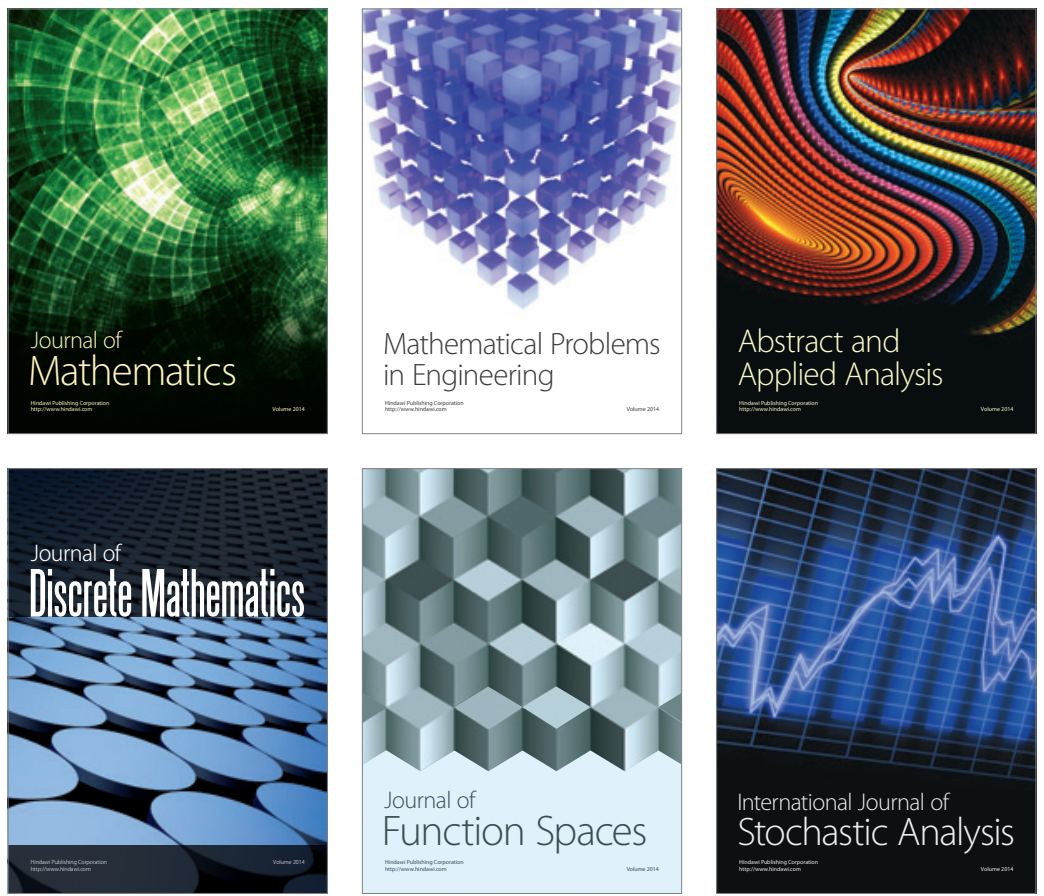

Journal of

Function Spaces

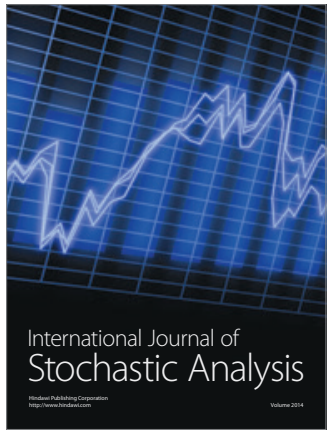

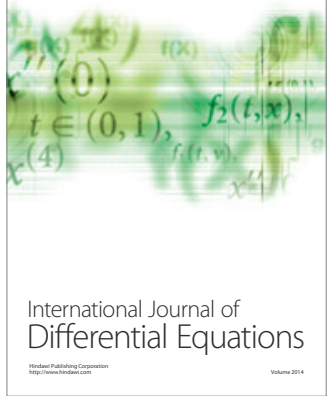
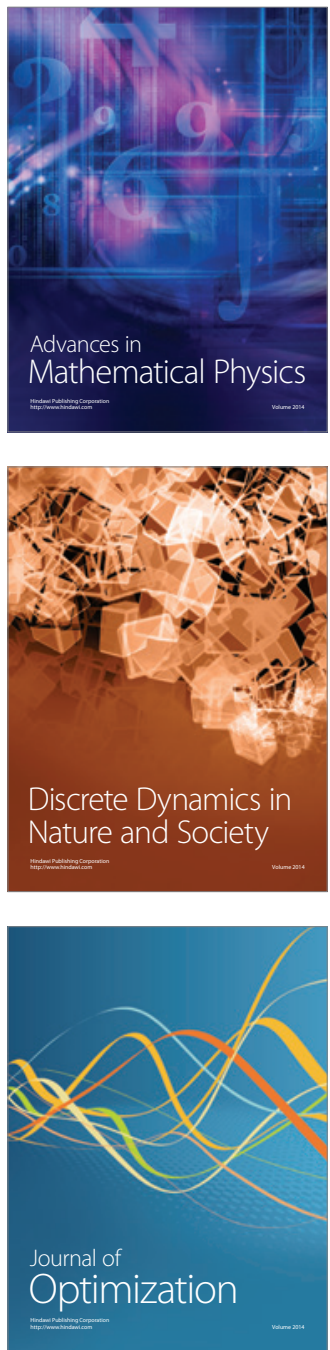